\title{
mBRAVE: The Multiplex Barcode Research And Visualization Environment
}

\author{
Sujeevan Ratnasingham ${ }^{\ddagger}$ \\ ‡ University of Guelph, Guelph, Canada
}

Corresponding author: Sujeevan Ratnasingham (sratnasi@uoguelph.ca)

Received: 05 Jul 2019 | Published: 10 Jul 2019

Citation: Ratnasingham S (2019) mBRAVE: The Multiplex Barcode Research And Visualization Environment. Biodiversity Information Science and Standards 3: e37986. https://doi.org/10.3897/biss.3.37986

\begin{abstract}
Widespread interest in the study of metabarcoding has resulted in data proliferation and the development of a multitude of powerful computational tools. Yet consistent and reproducible interpretation of the data remains challenging. The integration of different data types, software tools, and analytical parameters pose a barrier to scaling research. Further, though the majority of the necessary tools for performing these analyses are already implemented, there is limited support for high throughput analysis due to the requirement for heavy computational capacity. As a result of these complexities, many researchers lack the time, training, or infrastructure to work with larger datasets.
\end{abstract}

mBRAVE, the Multiplex Barcode Research And Visualization Environment, is a cloudbased data storage and analytics platform with standardized pipelines and a sophisticated web interface for transforming raw high-throughput sequencing (HTS) data into biological insights. mBRAVE integrates common analytical methods and links to the Barcode of Life Data (BOLD) System for reference datasets, presenting users with the ability to analyze large volumes of data, without requiring special technical training. mBRAVE's cloud architecture provides centralized and automated storage and compute capacity, thereby reducing the burden on individual researchers.

The mBRAVE platform seeks to alleviate the main informatic challenges faced by the metabarcoding research community: the storage and consistent interpretation of HTS data. It is now available for researcher use at www.mbrave.net. 


\section{Keywords}

metabarcoding, operational taxonomic units (otus), barcoding, bioinformatics

\section{Presenting author}

Sujeevan Ratnasingham

\section{Presented at}

Biodiversity_Next 2019

\section{Funding program}

Food From Thought (https://www.foodfromthought.ca/)

\section{Hosting institution}

Center For Biodiversity Genomics, University of Guelph 